ISSN 0103-9954

\title{
COMPARAÇÃO DE EQUAÇÕES VOLUMÉTRICAS AJUSTADAS COM DADOS DE CUBAGEM E ANÁLISE DE TRONCO
}

\section{COMPARATIVE VOLUME EQUATIONS ADJUSTED WITH DATA OF STEM MEASUREMENTS AND STEM ANALYSIS}

\section{Cláudio Thomas ${ }^{1}$ César Martins Andrade ${ }^{2}$ Paulo Renato Schneider ${ }^{3}$ César Augusto Guimarães Finger ${ }^{3}$ \\ RESUMO}

Este trabalho teve como objetivo comparar estimativas de volume com casca, obtidas com equações originadas partindo de dados da cubagem com casca e análise de tronco sem casca respectivamente. A base de informações para o desenvolvimento deste estudo foi obtida em povoamentos de Pinus taeda L. no município de Ponte Alta do Norte, Santa Catarina, sendo composta de duzentos e quarenta árvores cubadas com casca em diferentes idades, as quais foram selecionadas aleatoriamente e distribuídas por classe de diâmetro. Outra fonte de dados foi obtida com a análise de tronco sem casca de trinta árvores-amostra, distribuídas entre médias e dominantes. Foram testados modelos matemáticos para cada fonte de dados, afim de selecionar o de melhor ajuste e precisão, com base no critério de seleção do Valor Ponderado dos Escores dos Parâmetros Estatísticos (VP). O resultado do VP revelou a equação de Schumacher-Hall como mais adequada na estimativa do volume para as respectivas fontes de dados. A comparação entre as estimativas de volume dos modelos ajustados, partindo de dados da cubagem com casca e da análise de tronco sem casca, foi realizada com o teste Qui-quadrado, que demonstrou não haver diferença significativa entre elas, permitindo afirmar que é possível obter o volume com casca com base em uma equação gerada com dados de volume sem casca, obtidos pela análise de tronco.

Palavras-chave: volume individual; modelagem; regressão.

\begin{abstract}
This work aimed at comparing volume estimates with bark, obtained with equations originated from data of the stem measurements with bark and stem analysis without bark, respectively. The base of information for the development of this study was obtained in forest of Pinus taeda L. in the municipal district of Ponte Alta do Norte, Santa Catarina, being composed by two hundred and forty trees measured with bark in different ages, which were selected aleatorily and distributed by diameter class. Another source of data was obtained with the stem analysis without bark of thirty sample trees, distributed among averages and dominant. Mathematical models were tested for each source of data, in order to select the one of better adjustment and precision, with base in the selection criterion of the Score Pondered Value of the Statistical Parameters (VP). The result of VP revealed the equation of Schumacher-Hall as the most appropriated one in the estimate of the volume for the respective sources of data. The comparison between the estimates of volume of the adjusted models starting from data of the stem measurements with bark and of the stem analysis without bark was accomplished with the test Qui-square, which demonstrated that there area no significant differences to be significant difference among the them allowing to affirm that it is possible to obtain the volume with bark starting from an equation generated with volume data without bark, obtained through the stem analysis.
\end{abstract}

Keywords: individual volume; modeling; regression.

\section{INTRODUÇÃO}

A constante busca de alternativas que possibilitem otimizar a produção de florestas passa pelo aprimoramento de técnicas de biometria, inventário e manejo florestal.

1. Acadêmico do Curso de Graduação em Engenheira Florestal, Centro de Ciências Rurais, Universidade Federal de Santa Maria, CEP 97105-900, Santa Maria (RS). Bolsista PIBIC/CNPq. claudio4022002@yahoo.com.br

2. Acadêmico do Curso de Graduação em Engenheira Florestal, Centro de Ciências Rurais, Universidade Federal de Santa Maria, CEP 97105-900, Santa Maria (RS). Bolsista PIBIC/CNPq. cesarmartins@mail.ufsm.br

3. Engenheiro Florestal, Dr., Professor Titular do Departamento de Ciências Florestais, Centro de Ciências Rurais, Universidade Federal de Santa Maria, CEP 97105-900, Santa Maria (RS).paulors@ccr.ufsm.br

4. Engenheiro Florestal, Dr., Professor Adjunto do Departamento de Ciências Florestais, Centro de Ciências Rurais, Universidade Federal de Santa Maria, CEP 97105-900, Santa Maria (RS). finger@ccr.ufsm.br

Recebido para publicação em 17/01/2006 e aceito em 23/08/2006. 
O volume constitui uma das informações de maior importância para o conhecimento do potencial disponível em um povoamento florestal, haja vista que o volume individual fornece subsídios para a avaliação do estoque de madeira e análise do potencial produtivo das florestas.

Uma ferramenta amplamente utilizada nas estimativas e prognoses da produção madeireira é o emprego de equações volumétricas cujos parâmetros são determinados por regressão, constituindo um procedimento eficiente para a quantificação da produção em volume de um povoamento florestal (Parent apud Santana e Encinas, 2004). O procedimento de maior uso na estimativa do volume individual é o emprego de equações em que o volume é a variável dependente, associado a variáveis independentes de fácil mensuração na floresta, como o diâmetro à altura do peito e a altura (Machado et al., 2002).

Muitas equações matemáticas foram desenvolvidas para estimar o volume de povoamentos florestais, e apesar da eficiência de alguns modelos, estes nem sempre se ajustam a todas as espécies e condições, sendo recomendável testá-los e, por meio de estatísticas adequadas, identificar o melhor para cada caso.

Inúmeros pesquisadores ajustaram e compararam diversas funções matemáticas que expressam o volume, para diferentes espécies, sítios e regimes de manejo, entre eles: Silva et al. (1978), que construiram tabelas de volume comercial para Eucalyptus spp.; Schneider (1984), no cálculo do volume com casca para Pinus elliottii; Machado et al. (2002) modelaram o volume de Pinus oocarpa em diferentes idades e regimes de desbaste; Santana e Encinas (2004) desenvolveram equações para Eucalyptus saligna, entre outros.

Loetsch et al. (1973) consideram que, para a construção de uma tabela de volume local, se faz necessária a cubagem de 50 a 100 árvores; para áreas mais extensas, recomendam amostragem de centenas de árvores, visando a cobrir os diferentes sítios, idades, classes de diâmetro e formas de manejo a que a floresta tenha sido conduzida. Nesse caso, os custos envolvidos na obtenção dos dados de cubagem, para ajustar equações que são específicas para cada espécie, idade, ciclo ou região, são altos.

No intuito de facilitar a obtenção de dados utilizados para o ajuste de funções de volume, e conseqüentemente reduzir os custos do inventário, tem-se recorrido à utilização da dendrocronologia, também denominada análise de tronco, a qual trata da reconstituição do crescimento passado da árvore. Esta técnica considera cada ano de crescimento como uma árvore individual, permitindo a obtenção de um extenso conjunto de dados apropriados para ajustes de equações de volume, o que implica numa redução dos custos de obtenção de dados.

Nesse contexto, levando em consideração que a cubagem rigorosa consiste de um processo oneroso, e que a análise de tronco possibilita a obtenção de dados adequados para o ajuste de equações volumétricas de Pinus taeda L., pretende-se com este trabalho:

a) Testar modelos matemáticos que expressam o volume em função do diâmetro á altura do peito e altura para dados de volume com e sem casca, e selecionar a equação de melhor ajuste e precisão.

b) Estimar o volume de madeira com casca por meio de uma função gerada com dados de volume sem casca provenientes da análise de tronco, e compará-los aos volumes com casca obtidos pela equação originada com informações da cubagem.

\section{MATERIAL E MÉTODOS}

\section{Localização e caracterização da área de estudo}

Os dados utilizados no desenvolvimento deste trabalho foram obtidos em talhões de Pinus taeda L. pertencentes à empresa SEIVA S/A - Florestas e Indústrias, situada no município de Ponte Alta do Norte, região serrana de Santa Catarina, situada sob as coordenadas geográficas $27^{\circ} 07^{\prime}$ de latitude sul e $50^{\circ} 26^{\prime}$ de longitude oeste. A altitude do local é variável, ficando dentro das isométricas 800 e $1.200 \mathrm{~m}$ acima do nível do mar (Santa Catarina, 1986).

Segundo a classificação de Köppen, o clima da região é do tipo "Cfb", subtropical úmido com verões frescos, não apresentando estação seca definida. Dados obtidos na estação metereológica da empresa indicam uma precipitação média anual de $1.740 \mathrm{~mm}$ e temperatura média anual de $16,8^{\circ} \mathrm{C}$.

$\mathrm{O}$ solo da região é constituído dos tipos $\mathrm{TBHa} 2$ e Cha5, ambos caracterizados como cambissolo bruno húmico álico de textura argilosa, com face pedregosa e não-pedregosa (Santa Catarina, 1986). 
Os povoamentos, onde foram tomadas as árvore-amostra, diferiam quanto à idade, densidade e regime de manejo.

\section{Processo de amostragem e coleta de dados}

Inicialmente foram selecionadas aleatoriamente e cubadas 240 árvores, com idade variando de 8 a 28 anos, distribuídas em diferentes classes de diâmetros e sítios.

No cálculo do volume rigoroso, foi utilizado o método de Smalian, aplicando-se a seguinte equação geral: $v=v_{0}+\sum \frac{g_{i}+g_{i+1}}{2} \cdot l_{i}+g_{n} \cdot l_{n} \cdot \frac{1}{3}$

Em que: $v=$ volume total; $v_{0}=$ volume do toco; $g_{i}=$ área basal na i-ésima posição; $l_{i}=$ comprimento da seç̧ão na i-ésima posição; $g_{n}=$ área basal do cone; $l_{n}=$ comprimento do cone.

Também foram abatidas trinta árvores para a realização da análise de tronco, sendo esse grupo dividido em 15 árvores médias e 15 dominantes. Como critério de seleção da árvore média, foi considerado o indivíduo com diâmetro de área basal individual média aritmética da amostra. Para a seleção da árvore dominante, foi utilizado o conceito da altura dominante de Assmann, que considera a árvore com diâmetro médio aritmético das cem árvores mais grossas por hectare.

Para a realização da análise de tronco, foi utilizada metodologia apresentada por Finger (1992), tendo sido retiradas secções transversais do fuste, nas posições fixas de $0,30 \mathrm{~m}, 0,80 \mathrm{~m}$, dap, e partindo desta, a cada metro. Posteriormente essas secções foram secas, lixadas e levadas ao Laboratório de Crescimento e Produção Florestal da Universidade Federal de Santa Maria para a medição dos anéis de crescimento. Nesse processo, foi utilizado o aparelho Lintab II conectado ao Software TSAP Version 3.0. Para o processamento dos dados originados da análise de tronco e obtenção dos resultados de volume, foi utilizado o Software Anatron (Análise de tronco).

\section{Equações volumétricas}

Foram testados nove modelos matemáticos de volume selecionados na literatura florestal (Tabela 1), para dados com casca obtidos pela cubagem e dados sem casca provenientes da análise de tronco.

TABELA 1: Equações testadas para a estimativa do volume total com e sem casca, partindo de dados da cubagem e análise de tronco.

TABLE 1: Equations tested for the estimate of the total volume with and without bark, starting from data of the stem measurements and stem analysis.

\begin{tabular}{clc}
\hline Equações & \multicolumn{1}{c}{ Modelos } & Autores \\
\hline 1 & $v=b_{0}+b_{1} \cdot d^{2} \cdot h$ & Spurr \\
2 & $v=b_{0}+b_{1} \cdot d+b_{2} \cdot d^{2}$ & Hohenadl-Krenn \\
3 & $v=b_{0}+b_{1} \cdot d^{2}+b_{2} \cdot\left(d^{2} \cdot h\right)+b_{3} \cdot h$ & Stoate \\
4 & $v=b_{1} \cdot d^{2}+b_{2} \cdot\left(d^{2} \cdot h\right)+b_{3} \cdot\left(d \cdot h^{2}\right)+b_{4} \cdot h^{2}$ & Näslund \\
5 & $v=b_{0}+b_{1} \cdot d+b_{2} \cdot d^{2}+b_{3} \cdot(d \cdot h)+b_{4} \cdot\left(d^{2} \cdot h\right)$ & Meyer \\
6 & $v=b_{0}+b_{1} \cdot d+b_{2} \cdot d^{2}+b_{3} \cdot(d \cdot h)+b_{4} \cdot\left(d^{2} \cdot h\right)+b_{5} \cdot h$ & Meyer (Mod.) \\
7 & $\ln v=b_{0}+b_{1} \cdot \ln \left(d^{2} \cdot h\right)$ & Spurr \\
8 & $\ln v=b_{0}+b_{1} \cdot \ln d+b_{2} \cdot \ln h$ & Schumacher-Hall \\
9 & $\ln v=b_{0}+b_{1} \cdot \ln d+b_{2} \cdot \ln { }^{2} d+b_{3} \cdot \ln h+b_{4} \cdot \ln ^{2} h$ & I. B.W. Alemanha \\
\hline
\end{tabular}

Em que: $v=$ volume; $d=$ diâmetro à altura do peito; $h=$ altura total; $b_{0}, b_{1}, b_{2}, b_{3}, b_{4}, b_{5}=$ coeficientes; $\ln =\operatorname{logaritmo}$ neperiano. Fonte: Prodan (1968); Loetsch et al. (1973); Schneider (1984). Modelos ajustados pelo SAS.

\section{Critério de seleção dos modelos matemáticos}

A seleção do melhor modelo de regressão foi baseada nas seguintes estatísticas: Coeficiente de Determinação Ajustado $\left(\mathrm{R}^{2}{ }_{\mathrm{Aj}}\right.$ ), Erro-Padrão da Estimativa $\left(\mathrm{S}_{\mathrm{yx}}\right)$, Coeficiente de Variação $(\mathrm{CV} \%)$, Valor de $\mathrm{F}$ 
e Valor Ponderado dos Escores Estatísticos (VP).

Concomitantemente ao ajuste das equações de volume, foi realizada a análise gráfica de resíduos, para verificar a ocorrência ou não de tendenciosidade nas estimativas da variável dependente volume.

\section{Coeficiente de Determinação Ajustado $\left(\boldsymbol{R}^{2}{ }_{A j}\right)$}

O Coeficiente de Determinação $\left(\mathrm{R}^{2}\right)$ expressa a quantidade da variação total explicada pela regressão. Como o Coeficiente de Determinação cresce à medida que se inclui uma nova variável ao modelo matemático, foi utilizado, como critério, o Coeficiente de Determinação Ajustado $\left(\mathrm{R}_{\mathrm{Aj}}^{2}\right)$ para o número de coeficientes da equação, obtido pela expressão:

$$
R_{A j .}^{2}=R^{2}-\left[\frac{K-1}{N-K}\right] \cdot\left(1-R^{2}\right)
$$
observações.

Em que: $R_{A j}^{2}=$ Coeficiente de Determinação Ajustado; $K=$ número de coeficientes da equação; $N=$ número de

Por esse critério, quanto mais próximo de um for o valor do Coeficiente de Determinação, melhor será o ajuste da linha de regressão.

\section{Erro Padrão da Estimativa $\left(S_{y x}\right)$}

O Erro-Padrão da Estimativa indica a precisão do ajuste de um modelo matemático, e somente deve ser utilizado como comparador quando as variáveis dependentes apresentarem a mesma unidade de medida. Esse parâmetro é obtido pela fórmula:

$$
S_{y x}=\sqrt{Q M_{\text {res. }}}
$$

Em que: $S_{y x}=$ Erro-Padrão da Estimativa; $Q M_{\text {res. }}=$ Quadrado Médio do resíduo, obtido na análise da variância. equação.

Assim, quanto menor o Erro-Padrão da Estimativa, melhores são as estimativas obtidas com a

\section{Coeficiente de Variação (CV)}

Segundo Meyer (1941), o coeficiente de variação é uma importante opção para a comparação de equações com variáveis dependentes de diferentes unidades. Esse coeficiente na forma percentual é obtido pela fórmula:

$$
C V=\frac{S_{y x}}{\bar{y}} \cdot 100
$$

Em que: $C V=$ Coeficiente de Variação em percentagem; $S_{y x}=$ Erro-Padrão da Estimativa; $\bar{y}=$ média aritmética da variável dependente.

Dessa forma, quanto menor o valor do coeficiente de variação, melhor e mais precisa é a estimativa.

\section{Valor de F}

$\mathrm{O}$ valor de $\mathrm{F}$ calculado na análise da variância também foi utilizado como um dos parâmetros estatísticos na determinação do melhor modelo matemático. Por esse critério, quanto maior o valor de $\mathrm{F}$, melhor o ajuste da equação.

\section{Valor Ponderado dos Escores dos Parâmetros Estatísiticos (VP)}

Esse critério de seleção leva em consideração todas as variáveis estatísticas anteriormente descritas, e por isso sintetiza os resultados, facilitando o processo de seleção do melhor modelo matemático.

O Valor Ponderado foi determinado atribuindo-se valores ou pesos aos parâmetros estatísticos. As estatísticas foram ordenadas de acordo com a sua eficiência, sendo atribuído peso 1 para a equação mais eficiente, 2 para a segunda e assim sucessivamente (ranking), conforme metodologia descrita por Thiersch (1997). Após essa classificação individual, efetuou-se o somatório da pontuação para cada modelo matemático, sendo que a equação de volume que recebeu a menor soma foi recomendada como mais adequada para uso. 


\section{Distribuição dos resíduos}

Ao final do processo de seleção dos modelos matemáticos, foi analisada a distribuição gráfica dos resíduos, com o objetivo de verificar se o modelo selecionado pelo critério do Valor Ponderado apresentava alguma tendência na estimativa da variável dependente volume.

\section{Teste de paralelismo e coincidência das estimativas de volume}

No intuito de investigar a possibilidade de obter volume com casca, com base em uma equação gerada com dados de volume sem casca, provenientes da análise de tronco, foi aplicado o teste Qui-quadrado $\left(\chi^{2}\right)$, com um nível de significância $(\alpha)$ de 0,01 , utilizando-se a equação:

$$
\chi^{2}=\sum_{i=1}^{n} \frac{\left(v_{c}-v_{s}\right)^{2}}{v_{c}}
$$

Em que: $v_{c}=$ volume com casca estimado pela equação originada de dados da cubagem; $v_{s}=$ volume sem casca estimado pela equação originada da análise de tronco.

\section{RESULTADOS E DISCUSSÃO}

Nas Tabelas 2 e 3, são apresentados os coeficientes e parâmetros estatísticos dos modelos testados para a estimativa de volume com e sem casca.

TABELA 2: Parâmetros das equações testadas para ajustar o volume com casca, partindo de dados da cubagem.

TABLE 2: Parameters of the equations tested to adjust the volume with bark, starting from data of the stem measurements.

\begin{tabular}{|c|c|c|c|c|c|c|c|c|c|c|}
\hline \multirow[t]{2}{*}{ Equação } & \multicolumn{6}{|c|}{ Coeficientes } & \multirow{2}{*}{$\mathrm{R}^{2}{ }_{\mathrm{Aj}}$} & \multirow{2}{*}{$S_{x y}$} & \multirow{2}{*}{$\mathrm{CV} \%$} & \multirow{2}{*}{$\mathrm{F}$} \\
\hline & $\mathrm{b}_{0}$ & $b_{1}$ & $\mathrm{~b}_{2}$ & $b_{3}$ & $\mathrm{~b}_{4}$ & $\mathrm{~b}_{5}$ & & & & \\
\hline 1 & 0,05284 & 0,000035 & & & & & 0,9796 & 0,1600 & 14,49 & $11.594,5$ \\
\hline 2 & $-0,07775$ & $-0,00643$ & 0,00116 & & & & 0,9388 & 0,2777 & 25,13 & $1.848,2$ \\
\hline 3 & $-0,14672$ & 0,000015 & 0,000033 & 0,01173 & & & 0,9810 & 0,1540 & 13,94 & $4.184,3$ \\
\hline 4 & - & 0,000064 & 0,000029 & 0,000008 & 0,000049 & & 0,9862 & 0,1544 & 13,97 & $6.226,1$ \\
\hline 5 & $-0,03338$ & $-0,00219$ & 0,0000002 & 0,000429 & 0,000030 & & 0,9813 & 0,1536 & 13,89 & $3.158,4$ \\
\hline 6 & $-0,01360$ & $-0,00302$ & 0,0000008 & 0,000490 & 0,000029 & $-0,0014$ & 0,9812 & 0,1539 & 13,92 & $2.516,3$ \\
\hline 7 & $-10,0838$ & 0,98979 & & & & & 0,9922 & 0,1005 & $-23,17$ & $31.205,6$ \\
\hline 8 & $-10,2077$ & 1,87512 & 1,14843 & & & & 0,9929 & 0,0970 & $-20,98$ & $15.951,6$ \\
\hline 9 & $-10,3443$ & 1,91090 & $-0,00496$ & 1,20572 & $-0,01083$ & & 0,9924 & 0,0982 & $-22,41$ & $8.345,9$ \\
\hline
\end{tabular}

Em que: $\mathrm{R}_{\mathrm{Aj} .}^{2}=$ coeficiente de determinação ajustado; $\mathrm{S}_{\mathrm{xy}}=$ erro-padrão da estimativa; $\mathrm{CV}=$ coeficiente de variação em percentagem; $\mathrm{F}=$ valor de $\mathrm{F}$ da análise da variância; $b_{0}, b_{1}, b_{2}, b_{3}, b_{4}, b_{5}=$ coeficientes.

Os dados da Tabela 2, referentes aos parâmetros estatísticos obtidos pelo ajuste da equação de volume com casca partindo de dados da cubagem, revelam que os nove modelos testados apresentaram bom desempenho estatístico quanto aos valores $\mathrm{R}^{2}{ }_{\mathrm{Aj}}$., variando de 0,9388 a 0,9929 , o que demonstra um alto grau de ajuste de descrição da variável dependente (volume) pelas variáveis independentes (dap e altura).

O coeficiente de variação variou de $-23,17$ a $25,13 \%$, com valores de $F$ entre 1.848,2 e 31.205,6 altamente significativos.

De acordo com os parâmetros das equações testadas para ajustar o volume sem casca, partindo de dados de análise de tronco, é possível observar, na Tabela 3, que da mesma forma como visto para os dados

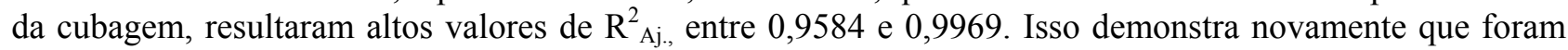
obtidas estimativas precisas com os modelos testados 
TABELA 3: Parâmetros das equações testadas para ajustar o volume sem casca, partindo de dados da análise de tronco.

TABLE 3: Parameters of the equations tested to adjust the volume without bark, starting from data of the stem analysis.

\begin{tabular}{|c|c|c|c|c|c|c|c|c|c|c|}
\hline \multirow[t]{2}{*}{ Equação } & \multicolumn{6}{|c|}{ Coeficientes } & \multirow{2}{*}{$\mathrm{R}_{\mathrm{Aj} .}^{2}$} & \multirow{2}{*}{$S_{x y}$} & \multirow{2}{*}{ CV\% } & \multirow{2}{*}{$\mathrm{F}$} \\
\hline & $\mathrm{b}_{0}$ & $\mathrm{~b}_{1}$ & $\mathrm{~b}_{2}$ & $\mathrm{~b}_{3}$ & $\mathrm{~b}_{4}$ & $\mathrm{~b}_{5}$ & & & & \\
\hline 1 & 0,03742 & 0,000037 & & & & & 0,9865 & 0,0885 & 11,77 & $57.844,9$ \\
\hline 2 & $-0,06078$ & $-0,01022$ & 0,00140 & & & & 0,9584 & 0,1555 & 20,67 & $9.102,2$ \\
\hline 3 & $-0,05634$ & $-0,00016$ & 0,0000392 & 0,00896 & & & 0,9894 & 0,0785 & 10,44 & $24.585,1$ \\
\hline 4 & - & 0,000342 & 0,0000035 & 0,000045 & $-0,00052$ & & 0,9901 & 0,0729 & 9,43 & $22.554,4$ \\
\hline 5 & $-0,10033$ & 0,010413 & $-0,00047$ & 0,000282 & 0,000041 & & 0,9905 & 0,0743 & 9,91 & $20.679,9$ \\
\hline 6 & 0,02940 & 0,01397 & $-0,000694$ & 0,00148 & 0,000031 & $-0,0281$ & 0,9917 & 0,0693 & 9,22 & $18.935,3$ \\
\hline 7 & $-9,91573$ & 0,97762 & & & & & 0,9963 & 0,0744 & $-8,57$ & $213.810,0$ \\
\hline 8 & $-9,84274$ & 1,81743 & 1,10282 & & & & 0,9969 & 0,0710 & $-8,19$ & $117.125,0$ \\
\hline 9 & $-9,87232$ & 2,56300 & $-0,11694$ & 0,26032 & 0,15122 & & 0,9966 & 0,0726 & $-7,79$ & $64.632,0$ \\
\hline
\end{tabular}

Em que: $\mathrm{R}_{\mathrm{Aj}}^{2}=$ coeficiente de determinação ajustado; $\mathrm{S}_{\mathrm{xy}}=$ erro-padrão da estimativa; $\mathrm{CV}=$ coeficiente de variação em percentagem; $\mathrm{F}=$ valor de $\mathrm{F}$ da análise da variância; $b_{0}, b_{1}, b_{2}, b_{3}, b_{4}, b_{5}=$ coeficientes.

Com relação ao $S_{y x}$, os valores variaram de 0,0693 a 0,1555 . O menor coeficiente de variação foi de 8,57\% para a equação 9, e o valor de $\mathrm{F}$ mais significativo foi de $213.810,0$ para o modelo 7 .

A seleção dos modelos com base na proposta de Thiersch (1997), que considera o Valor Ponderado obtido pelos escores de cada estatística tomadas em conjunto, é apresentada nas Tabelas 4 e 5 respectivamente, para as equações desenvolvidas com dados de árvores cubadas com casca e para árvores de análise de tronco sem casca respectivamente.

TABELA 4: Valor Ponderado dos Escores dos Parâmetros Estatísticos para as equações de volume com casca, testadas partindo de dados da cubagem.

TABLE 4: Score Pondered Value of the Statistical Parameters for the volume equations with bark, tested from data of the stem measurements.

\begin{tabular}{c|c|c|c|c|c}
\hline \multirow{2}{*}{ Equação } & \multicolumn{4}{|c|}{ Escores dos Parâmetros Estatísticos } & \multirow{2}{*}{$\begin{array}{c}\text { Valor } \\
\text { Ponderado (VP) }\end{array}$} \\
\cline { 2 - 5 } & $\mathrm{R}_{\mathrm{Aj}}^{2}$ & $\mathrm{~S}_{\mathrm{xy}}$ & $\mathrm{CV} \%$ & $\mathrm{~F}$ & 24 \\
2 & 8 & 8 & 5 & 3 & 36 \\
3 & 9 & 9 & 9 & 9 & 22 \\
4 & 7 & 6 & 3 & 6 & 20 \\
5 & 4 & 7 & 4 & 5 & 17 \\
6 & 5 & 4 & 1 & 7 & 21 \\
7 & 6 & 5 & 2 & 8 & 15 \\
8 & 3 & 3 & 8 & 1 & 10 \\
9 & 1 & 1 & 6 & 2 & 15 \\
\hline
\end{tabular}

Em que: $\mathrm{R}_{\mathrm{Aj}}^{2}=$ coeficiente de determinação ajustado; $\mathrm{S}_{\mathrm{xy}}=$ erro-padrão da estimativa; $\mathrm{CV}=$ coeficiente de variação em percentagem; $F$ = valor de $F$ da análise da variância. 
TABELA 5: Valor Ponderado dos Escores dos Parâmetros Estatísticos para as equações de volume sem casca, testadas partindo de dados da análise de tronco.

TABLE 5: Score Pondered Value of the Statistical Parameters for the volume equations without bark, tested from data of the stem analysis.

\begin{tabular}{c|c|c|c|c|c}
\hline \multirow{2}{*}{ Equação } & \multicolumn{4}{|c|}{ Escores dos Parâmetros Estatísticos } & \multicolumn{2}{c}{$\begin{array}{c}\text { Valor } \\
\text { Ponderado (VP) }\end{array}$} \\
\cline { 2 - 5 } & $\mathrm{R}^{2}{ }_{\mathrm{Aj}}$ & $\mathrm{S}_{\mathrm{xy}}$ & $\mathrm{CV} \%$ & $\mathrm{~F}$ & 28 \\
2 & 8 & 8 & 8 & 4 & 36 \\
3 & 9 & 9 & 9 & 9 & 26 \\
4 & 7 & 7 & 7 & 5 & 21 \\
5 & 6 & 4 & 5 & 6 & 23 \\
6 & 5 & 5 & 6 & 7 & 17 \\
7 & 4 & 1 & 4 & 8 & 13 \\
8 & 3 & 6 & 3 & 1 & 7 \\
9 & 1 & 2 & 2 & 2 & 9 \\
\hline
\end{tabular}

Em que: $\mathrm{R}_{\mathrm{Aj}}^{2}=$ coeficiente de determinação ajustado; $\mathrm{S}_{\mathrm{xy}}=$ erro-padrão da estimativa; $\mathrm{CV}$ = coeficiente de variação em percentagem; $\mathrm{F}=$ valor de $\mathrm{F}$ da análise da variância.

O ranking do Valor Ponderado (VP), apresentado nas Tabelas 4 e 5, atribuiu valores de 1 a 9 para cada parâmetro estatístico considerado $\left(\mathrm{R}_{\mathrm{Aj}}^{2}, \mathrm{~S}_{\mathrm{xy}}, \mathrm{CV} \%, \mathrm{~F}\right)$, sendo designado $1 \mathrm{o}$ melhor e 9 para o pior. A função número 8, de Schumacher-Hall, obteve o menor Valor Ponderado para as duas fontes de dados, com casca e sem casca, sendo então a selecionada para as estimativas de volume.

Nas Figuras 1 e 2, são apresentados os gráficos dos resíduos do volume para os dois conjuntos de dados, não demonstrando tendenciosidade de ajuste da variável dependente volume.

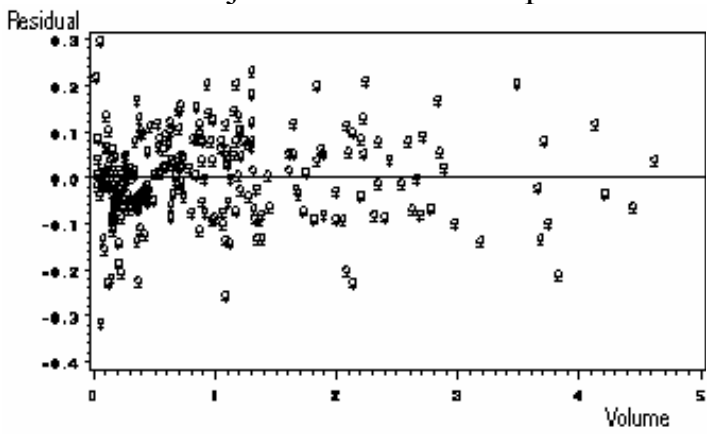

FIGURA 1: Distribuição dos resíduos para o volume com casca estimado pela equação ajustada com dados da cubagem.

FIGURE 1: Distribution of the residues for the volume with bark considered for the adjusted equation with data of stem measurements.

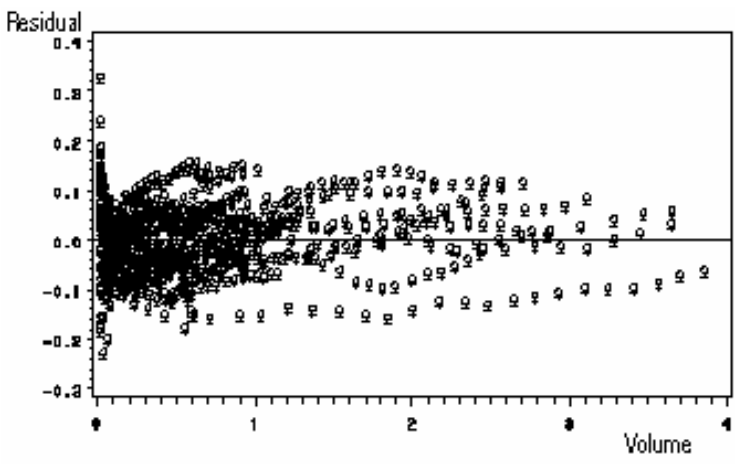

FIGURA 2: Distribuição dos resíduos para o volume sem casca estimado pela equação ajustada com dados da análise de tronco.

FIGURE 2: Distribution of the residues for the volume without bark considered for the adjusted equation with data of the stem analysis. 
O teste Qui-quadrado $\left(\chi^{2}\right)$, aplicado para comparar os volumes calculados com as equações ajustadas, mostrou não haver diferença significativa para a probabilidade de 0,01 . O valor de $\chi^{2}$ calculado foi igual a 0,0008 , menor que o tabelado de 23,21 , assim, se pode afirmar que as duas curvas não diferem estatisticamente a essa probabilidade.

Esse resultado estatístico pode ser observado na Figura 3, que representa graficamente a tendência das estimativas de volume, obtidas por equações de volume ajustadas com dados oriundos da cubagem com casca e análise de tronco sem casca, sendo possível verificar a quase-total sobreposição das duas curvas de volume.

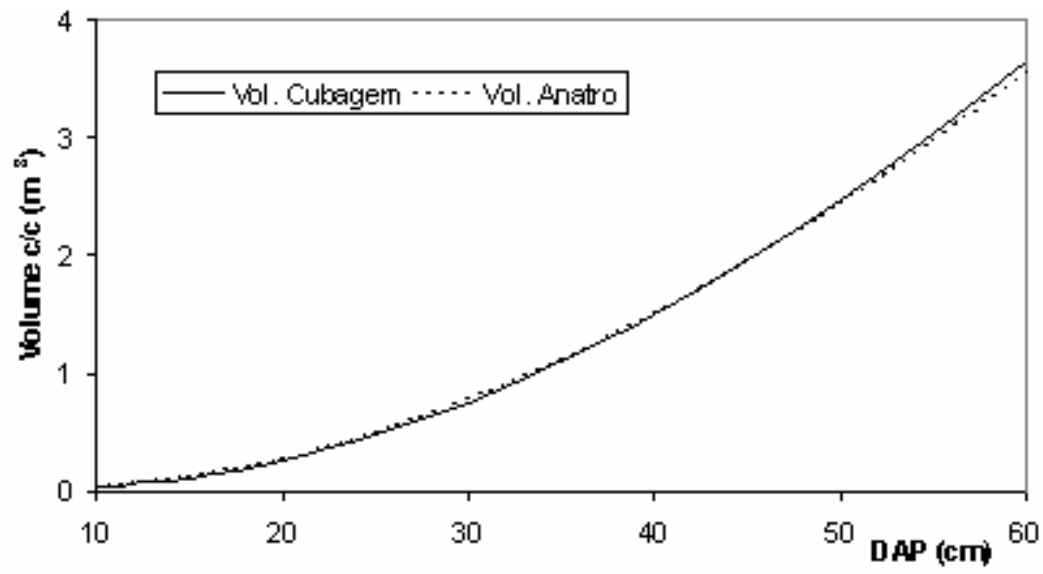

FIGURA 3: Curvas de volume com casca obtidas pelas equações ajustadas com dados de cubagem e análise de tronco.

FIGURE 3: Volume curves with bark obtained through adjusted equations with stem measurements data and stem analysis.

\section{CONCLUSÕES}

A análise do Valor Ponderado, juntamente com a observação gráfica dos resíduos resultantes do ajuste de equações de volume, permitem concluir que para o conjunto de dados utilizados, o modelo de Schumacher-Hall apresenta o melhor desempenho nas estimativas de volume total com e sem casca, para dados oriundos de cubagem e análise de tronco.

O teste Qui-quadrado revelou que não houve diferença significativa nas estimativas das duas equações ajustadas com dados da cubagem com casca e análise de tronco sem casca, respectivamente, demonstrando que é possível obter com precisão, informações de volume com casca partindo de uma equação gerada com dados de volume sem casca.

\section{REFERÊNCIAS BIBLIOGRÁFICAS}

FINGER, C. A. G. Fundamentos de biometria florestal. Santa Maria: UFSM, 1992. 269p.

LOETSCH, F.; ZÖHRER, F.; HALLER, K. E. Forest inventory. Reinbek: Federal Research Organization for Forestry and Forest Products, Forest Inventory Section, 1973. 469p. v. 2.

MACHADO, S. A.; CONCEIÇÃO, M. B.; FIGUEIREDO, D. J. Modelagem do volume individual para diferentes idades e regimes de desbaste em plantações de Pinus oocarpa. Ciências Exatas e Naturais, Curitiba, v. 4, n. 2, p. 185196, 2002.

MEYER, H. A. A correction for a systematic errors occurring in the application of the logarithmic volume equation. Pensylvania: Forestry School Research, 1941. 15p. (Forestry School Research Paper; 7)

PRODAN, M. Forest biometrics. New York: Pergamon Press, 1968. 447p.

SANTA CATARINA. Gabinete de Planejamento e Coordenação Geral. Sub-chefia de Estatística, Geografia e Informática. Atlas de Santa Catarina. Rio de Janeiro: Aerofoto Cruzeiro, 1986. 176p.

SANTANA, O. A.; ENCINAS, J. I. Equações volumétricas para uma plantação de Eucalyptus urophylla destinada a produção de lenha. In: SIMPÓSIO LATINO-AMERICANO SOBRE MANEJO FLORESTAL, 3. Anais... Santa Maria: UFSM/PPGEF, 2004. p. 107-111. 
SILVA, J. A. A.; PAULA NETO, F.; BRANDI, R. M. Análise de modelos volumétricos para a construção de tabelas de volume comercial para Eucalyptus spp, segundo a espécie, região e métodos de regeneração. Revista Árvore, Viçosa, v. 2, n. 1, p. 86-89,1978.

THIERSCH, A. A eficiência das distribuições diamétricas para prognose da produção de Eucalyptus camaldulensis. Lavras, 1997. 155f. Dissertação (Mestrado em Engenharia Florestal) - Universidade Federal de Lavras, Lavras, 1997. 